\title{
Computer simulation of the aggregates formation during flocculation process
}

\author{
Yurii Rybachuk ${ }^{1, *}$, and Andrzej Jodłowski ${ }^{1}$ \\ ${ }^{1}$ Lodz University of Technology, Department of Building, Architecture and Environment \\ Engineering, 90924 Lodz, 6 Politechniki Ave., Poland
}

\begin{abstract}
The main goal of this paper is to analyse physical and chemical aspects affecting the structure and strength of flocs, which are created during coagulation and flocculation of water impurities, from the point of view of process computer simulation. Proposed mathematical dependencies were used for computer modelling of the process as well as visualisation of the obtained results. The results of algorithms operation and visualization were shown as graphical representation. Laboratory studies were carried out to check the realism of the proposed algorithm.
\end{abstract}

\section{Introduction}

Surface water is frequently used for water supply purposes. Surface water usually contains pollution of various kinds, including colloidal and high dispersive matter. Colloidal particles are the most difficult to remove from water, because of their stable condition [1, 2]. Particles removal from water without their destabilization gives a negligible effect of water purification. Destabilization of particles can be achieved by chemical coagulation which is a very complex process due to the influence of many physical and chemical factors as: $\mathrm{pH}$ of water, $\zeta$-potential of the particle, dosage of the reagent and its type, reaction time and mixing speed, temperature, etc. Existing basic theories, based mainly on Smoluchowski's rapid coagulation principle, allow to examine the kinetics of the coagulation process and to predict effect of aggregation expressed as the particles number reduction [3]. Further development of basic theories allow to create mathematical models of flocculation, which consider the process in greater details, namely: characterize size, strength, density of resulting flocs [4]. It is also necessary to mention that newest models take into consideration a mechanism of flocs destruction during flocculation.

Quick development of the computer technology allows to implement sophisticated algorithms for the interpretation of existing theories and mathematical models, as well as visualization (based on fractal theory) [5-7]. Mathematical models and algorithms for simulation and visualization are used enabling to obtain results in $2 \mathrm{D}$ space. The disadvantages of these algorithms include the application of predefined number of primary objects (spheres) taking a part in the structure of aggregates.

The main goal of this paper is to apply mathematical model of the flocculation process and algorithms which allow to predict strength, mass and volume of primary particles

\footnotetext{
* Corresponding author: jrybachuck@gmail.com
} 
aggregates. Proposed algorithm was applied to computer program, which allowed to perform simulation using selected initial data. The second goal was to elaborate algorithm of aggregates visualization, which allows to describe shape and size of floc in a 3D space. The third goal of the work was to check the realism of the proposed model on the basis of laboratory studies.

\section{Flocculation process modelling}

\subsection{Main dependencies}

Analysis of the flocculation process taking into consideration the forces that affect particles aggregation, as well as forces acting in the reaction tank, allows to simulate aggregates formation in 3D space for the selected parameters of coagulation. Forces that affect the aggregation of colloidal particles can be expressed as equation showing balance of forces which are changed during the course of the process. Balance force $\left(F_{b a l}\right)$ can be used as the indicator of the strength of created aggregates. Coulomb force $\left(F_{C o}\right)$, van der Waals force $\left(F_{v d W}\right)$, Archimedes's force $\left(F_{A}\right)$, centrifugal force $\left(F_{c}\right)$ and the force of gravity $\left(F_{g}\right)$ have been included into equation (1).

$$
\frac{d F_{b a l}}{d t}=\frac{d F_{c o}}{d t}+\frac{d F_{v d W}}{d t}-\frac{d F_{A}}{d t}-\frac{d F_{c n}}{d t}-\frac{d F_{g}}{d t}
$$

The balance equation takes into account the forces, which can be divided into two groups - aggregation forces and destruction forces. A positive value of the balance forces indicates an increase of aggregates size and mass, while negative forces value show destruction of aggregates corresponding to their reduction in size and mass.

Coulomb force $\left(F_{C o}\right)$ refers to the force which allows for the aggregation and in the case of repulsion can cause destruction of floc and can be expresed as:

$$
F_{c o}=k \frac{\left|q_{+}\right|\left|q_{-}\right|}{r^{2}}
$$

where $q_{+}$is the charge of coagulant particle (C), $q_{-}$is the polution particle charge (C), $k$ is the coefficient of proportionality of this system $\left(\mathrm{N} \mathrm{m}^{2} / \mathrm{C}^{2}\right)$, and $r$ is the distance between particles $(\mathrm{m})$. The charge of the particle can be expressed taking into account dielectric permittivity $\left(d_{P}\right)$, $\varsigma$ potential of the particle, and radius of the particle $\left(r_{c}\right)$ according to the equation (3):

$$
q=\frac{d_{p} \zeta r_{c}}{k}
$$

The main parameter in this equation is the value of $\zeta$ potential. Its value is totally dependent on the water $\mathrm{pH}$ as was shown during the laboratory studies [8]. To maximize the attraction between the particles it is necessary to create such conditions, which allow to obtaine polution and reagent particles with different electric charge and its values to get maximal available value of attractive force.

The value of $r_{c}$ can be calculated according to the equation (4), when molar mass of substances $M(\mathrm{~g} / \mathrm{mol})$, Avogadro's number $N a\left(\mathrm{~mol}^{-1}\right)$ and particle densidy $\rho\left(\mathrm{kg} / \mathrm{m}^{3}\right)$ are used. 


$$
r_{c}=\frac{3 M}{4 \pi N_{a} \rho}
$$

Van der Waals forces $\left(F_{v d W}\right)$ occure at short distances between particles $\left(10^{-9} \mathrm{~m}\right)$. They can be expressed according to equation (5), where Hamaker constant $A(\mathrm{~J})$, the size of the particle $a(\mathrm{~m})$, and distance between the particle $H(\mathrm{~m})$ :

$$
F_{v d W}= \pm \frac{A a}{12 H^{2}}
$$

Their values are very difficult to calculate so values of $10^{-8}$ and $10^{-9} \mathrm{~N}$ were used for the calculation according to the results of experiments conducted by atomic force microscopes [9]. It is also necessary to take into account the repulsion between the particles, which can be caused by the spatial arrangement of dipoles with simple electric charge. The probability of such an arrangement of elements in which there is a repulsion can be defined from total value of polution and coagulant particles in reaction vessel. Van der Waals forces can take a negative value leading to floc destruction in the case of repulsion.

Other forces that affect the floc as destructive part are: Archimedes' force $\left(F_{A}\right)$ centrifugal force $\left(F_{c n}\right)$ in the aggitaed system, and gravitational force $\left(F_{g}\right)$ (equations 6 , 7 and 8):

$$
\begin{aligned}
& F_{A}=\rho_{w} g \Delta m_{a} / \rho_{a} \\
& F_{c n}=\Delta V^{2} m_{s} / \Delta r_{d} \\
& F_{g}=\Delta m_{a} g
\end{aligned}
$$

where $\rho_{w}$ is water density $\left(\mathrm{kg} / \mathrm{m}^{3}\right), g$ is graviatational acceleration $\left(\mathrm{m} / \mathrm{s}^{2}\right), \Delta m_{a}$ is aggregate's mass $(\mathrm{kg}), \rho_{a}$ is density of agregate $\left(\mathrm{kg} / \mathrm{m}^{3}\right), \Delta \mathrm{V}$ is velocity of water at a certain spatial point $(\mathrm{m} / \mathrm{s}), m_{s}$ is mass of stirrer $(\mathrm{kg}) ; \Delta r_{d}$ is a distance between stirrer and point of simulation $(\mathrm{m})$.

\subsection{Algorithm of the simulation}

The basic equation (1) contains the differential variables, namely: mass of floc, water velocity and distance from the stirrer to a point of simulation. The mass is changing in time, and the water velocity depends not only on time, but also on the spatial point of modeling (from the bias point of simulation in 3D space). The main algorithm is iterative in nature. Iterations time step goes from the 0 to the value of the time of flocculation (slow mixing time). It was also necessary to describe changes of mass, distance, and velocity of aggregates for each iteration. The subalgorithms were described for this purposes.

\subsubsection{Mass determination algorithm}

The initial mass of the floc is equal to the value of the mass of one particle of water pollutant. The weight of the floc may increase as a result of the particles attraction (sticking) and also in the case when the external forces acting on aggregates exceeds the force value, which causes agreggation of particles, floc's mass may decrease. The main issue is the determination of the amount of connective or detaching reagent and pollution particles for one iteration. The time incremination for one iteration is equal to $0.1 \mathrm{~s}$, which 
allows to minimize the amount of connective or detaching particles regarding the results obtained during laboratory studies. The number of interactive particles for 1 iteration can be determined by the number of neutralization $\left(N_{\text {neu }}\right)$ :

$$
N_{\text {neu }}=\frac{\left|q_{+}\right|}{\left|q_{-}\right|}
$$

The number of neutralization shows the number of particles of pollution, which can be connected by one particle of reagent, also it shows the mass growth:

$$
\begin{gathered}
m_{\text {new }}^{\text {floc }}=m_{\text {previos }}^{\text {floc }}+N_{\text {neu }} \cdot m_{\text {pollution }}+m_{\text {coagulant }}, F_{\text {bal }} \geq 0 \\
N_{\text {new }}^{\text {particles }}=N_{\text {previos }}^{\text {particles }}+N_{\text {neu }}+1, F_{\text {bal }} \geq 0 \\
m_{\text {new }}^{\text {floc }}=m_{\text {previos }}^{\text {floc }}-N_{\text {neu }} \cdot m_{\text {pollution }}-m_{\text {coagulant }}, F_{b a l}<0 \\
N_{\text {new }}^{\text {particles }}=N_{\text {previos }}^{\text {particles }}-N_{\text {neu }}-1, F_{\text {bal }}<0
\end{gathered}
$$

It is also necessary to check the plausibility of the obtained floc size. It is possible to compare the lenght of Kolmogorov microscale with obtained floc size [10] in the case when the exceeding of the value of microscale destruction occures. This operation can be performed in the end of whole algorithm. The rate of destruction defines randomly.

\subsubsection{Algorithm of the description of spatial point of simulation}

Different centrifugal force acts on floc when the aggrecate moves in the mixing tank. The value of centrifugal force depends on the water velocity at the point of the simulation and the distance of this point from the stirrer. The operation of this algorithm can be divided into two tasks. The first one is to determine velocity values at any point in the mixing tank and the second one is the simulation of the movement of floc (aggregate) in the reaction tank. The task of water velocity determination at any point of the tank can be solved by the solution of the Navier-Stokes equations for this tank [11].

Determination of the spatial point during modeling is solved as a result of the first problem solution. The starting point of modeling is defined by randomization. The definition of consecutive point is committed according to the definition of floc movement in space. The displacemeny of the aggregate is obtaned for the iteration increment $t(0.1 \mathrm{~s})$ :

$$
S=V t
$$

Since an iterative increment is a very small value, the velocity $(V)$ is the instantaneous velocity, which can be taken as a constant at the site between the two points. Knowing the coordinates of the current point and the value of the displacement to the next step of simulation it is possible to determine the coordinates of the following points of modeling:

$$
S=\sqrt{\left(x_{2}-x_{1}\right)^{2}+\left(y_{2}-y_{1}\right)^{2}+\left(z_{2}-z_{1}\right)^{2}}
$$

where $x_{1}, y_{1}, z_{1}$ - coordinates of current point of simulation; and $x_{2}, y_{2}, z_{2}$ - coordinates of next point of simulation.

Therefore, it is necessary to select such values $x_{2}, y_{2}, z_{2}$ to the value of $\mathrm{S}$ is calculated by the formula (13) to obtain the same value calculated by the formula (12). 


\subsubsection{Main algorithm and visualization algorithm}

Fig. 1 shows the connection of previously discussed algorithms to one main algorithm. A comlete statistics, which includes the coordinates of the displacement of particles in each iteration; the weight and volume of floc; the magnitude of the forces acting on the floc at each iteration; and the number of particles forming the aggregate can be obtained as the result of algorithm performance.

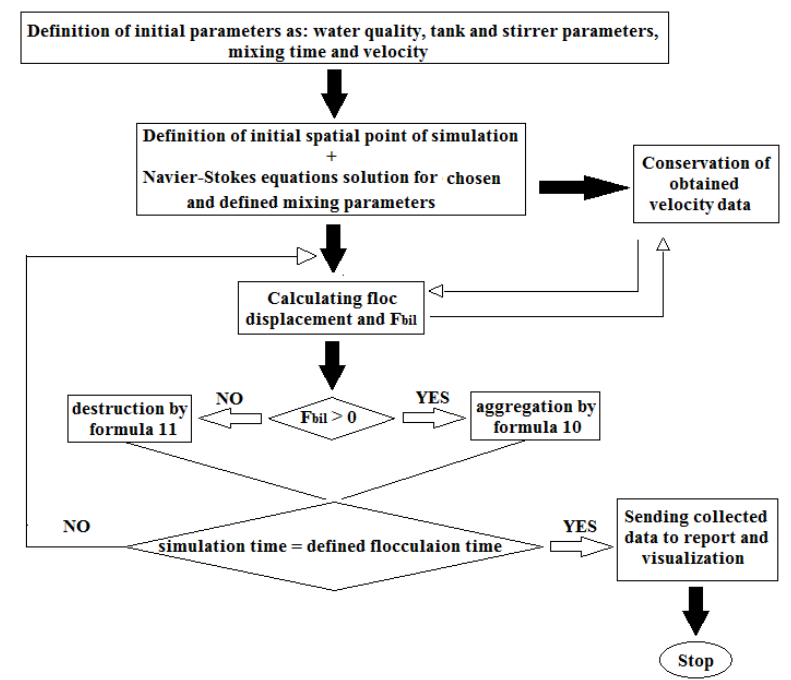

Fig. 1. Structure of the main simulation algorithm.

Knowing the number of particles forming the floc, it is possible to make visualization. The visualization can be implemented using randomization since the bonding of particles is totally random process and can not be described by any rules. The visualization algorithm is presented in Fig. 2.

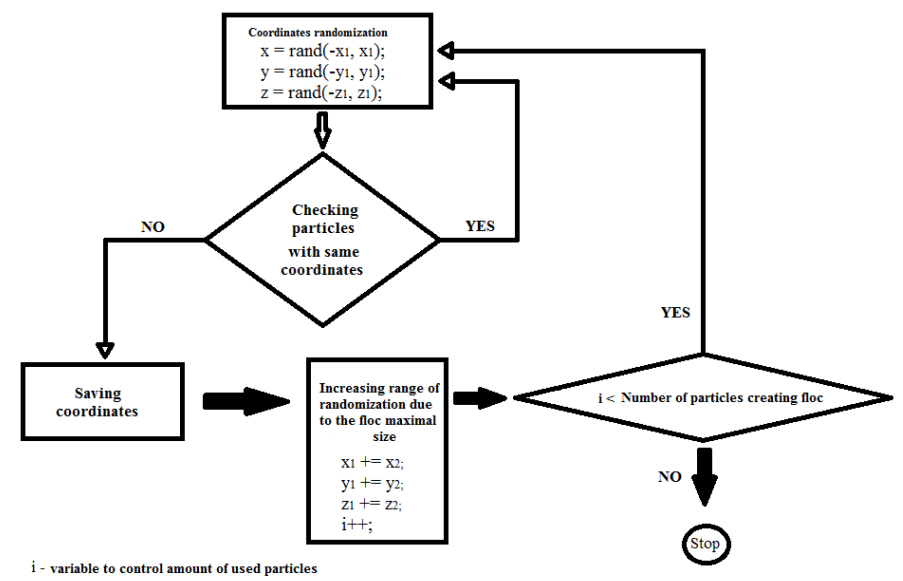

Fig. 2. Structure of the visualization algorithm. 


\section{Materials and methods}

Library for plots building was written that represents the implementation of the $\mathrm{C}++$ programming language, OpenGL graphics library and Qwt plots library to the translation of proposed algorithms to a computer program.

The following parameters were used for flocculation process simulation: the color of water $-64.2 \mathrm{mg} \mathrm{Pt} / \mathrm{L}, \mathrm{pH} 7, \zeta$ potential of the particles of pollution $-0.014 \mathrm{~V}$, the velocity and time of flocculation were $40 \mathrm{rpm}$ for 5 minutes. Applied parameters of the stirrer were: simple rectangle shape of the paddle radius was $0.1 \mathrm{~m}$, the height was $0.1 \mathrm{~m}$, the distance from the stirrer to the bottom of the tank was $0.2 \mathrm{~m}$. The parameters of the tank: height $0.5 \mathrm{~m}$, diameter $-0.4 \mathrm{~m}$. Alum $\left(\mathrm{Al}_{2}\left(\mathrm{SO}_{4}\right)_{3} \cdot 18 \mathrm{H}_{2} \mathrm{O}\right)$ at the concentration $10 \%$ was used as the coagulant, and $\zeta$ potential of the value of $+0.043 \mathrm{~V}$ was applied for the analysis.

The proposed algorithm of the simulation was also checked for feasibility during the labratory studies.

Humic acids extract obtained as a result of garden peat extraction at the concetration of $3.7 \mathrm{~cm}^{3} / \mathrm{L}$ created the pollution of water used during the laboratory investigation. That allowed to obtain water of the colour of $64.2 \mathrm{mg} \mathrm{Pt} / \mathrm{L}$ and turbidity of 2,14 NTU, what simulates real surface waters quality.

Aluminum sulfate $\left(\mathrm{Al}_{2}\left(\mathrm{SO}_{4}\right)_{3} \cdot 18 \mathrm{H}_{2} \mathrm{O}\right.$ at the concentation of $10 \%$ was used as coagulat. The $\mathrm{pH}$ was adjusted to $\mathrm{pH} 6.0 \pm 0.1 \mathrm{using} \mathrm{HCl}$ and $\mathrm{NaOH}$ solutions. The coagulant dose was set as $40 \mathrm{mg} \mathrm{Al} / \mathrm{L}$. The Velp Scientifica JLT-6 multi-site agitator with paddle agitators measuring $75 \times 25 \mathrm{~mm}$ was used during the studies. Reaction vessels in the form of polyethylene beakers had an active height of $130 \mathrm{~mm}$ and a diameter of $100 \mathrm{~mm}$. Rapid mixing was carried out for 1 minute at $100 \mathrm{rpm}$, followed by slow mixing for 10 minutes at a rotational speed of $10 \mathrm{rpm}$.

Malvern's Morphologi G3 image microscopic analyzer was used during the studies on the morphology of created aggregates (flocs). The analyzer's measuring system included an automatic microscope and a computer with software enabling the management of measurements and analysis of the obtained results.

\section{Results and discussion}

Fig. 3 shows graphical representation of results obtained during algorithm performance with the previously described parameters. It shows the trajectory of movement of the floc during $5 \mathrm{~min}$ at iterative time step of 0.1 second, which is 3000 iterations.

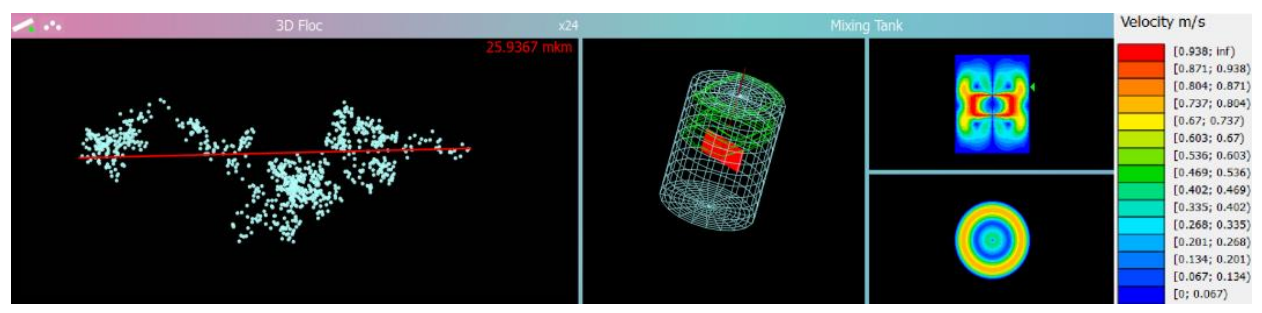

Fig. 3. Screenshots of program, which representing performed floc formation simulation.

Curve of the aggregate movement and acting forces were built and calculated using data taken from solution of the Navier-Stokes equations with SIMPLE method. In this example, presented floc, which consists of 853 particles, where pollution particles -514 , and the numebr of coagulant particles -339 . Floc has a relatively large size $-25.94 \mu \mathrm{m}$ as can be 
seen from Fig. 3. It is also possible to collect data about each iteration for further analysis of chosen parameters of flocculation. Such kind of availiable report is presented in Fig. 4.

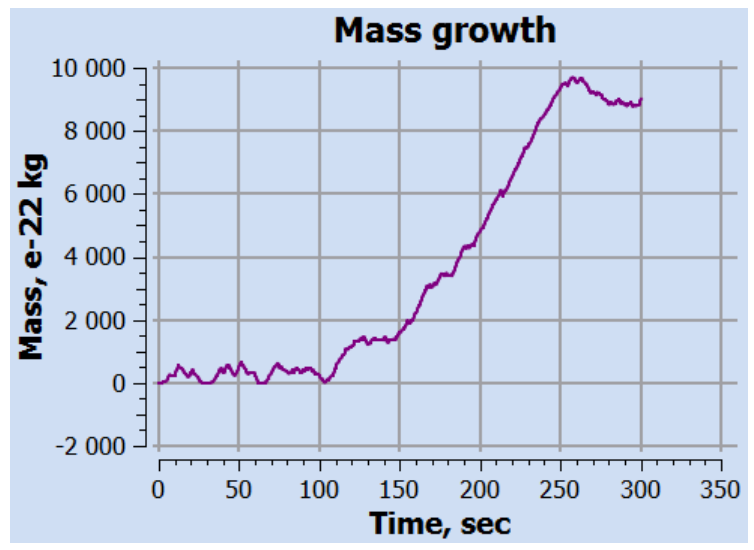

Fig. 4. Screenshot of program, which representing kinetics of floc formation in a time flow.

The influence of mixing forces on the floc formation can be seen in Fig. 4. Analysing the trajectory of floc movement, it wolud be able to notice that destruction takes place in areas near to stirrer's blades, where water has the highest velocity according to data obtained from Navier-Stokes equation.

The size distribution of aggregates obtained during the laboratory studies was similar to results obtained as a result of a number of computer simulations. Fig. 5 shows that increasing of stirrer velocity leads to greater impact of destructive forces and as the result small floc sizes. The similarity of model to this phenomenon was also obtained. Applied model shows that coagulation-flocculation cannot be performed or performing poorly, when $\mathrm{pH}$ values are in the range less than 5 or above 9. It happens because of the similar $\zeta$ potential sign (at $\mathrm{pH}>9$ ), as the result repulsion of particles occurs.
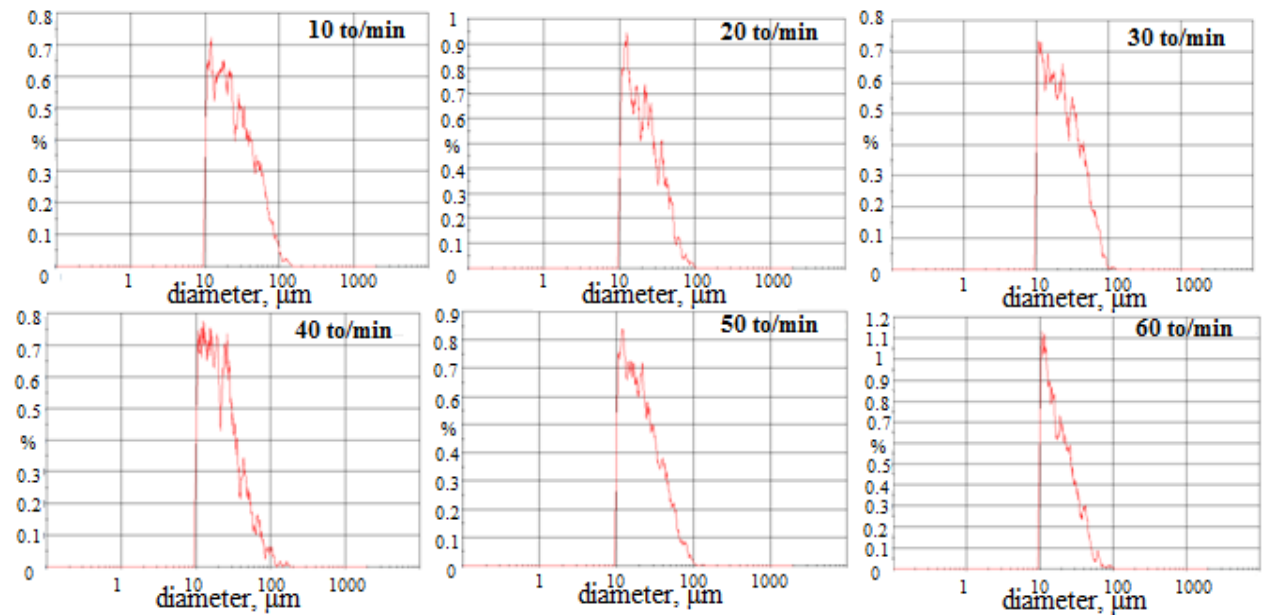

Fig. 5. Microscopy analysis of flocs distribution in stirred tank using different velocity of slow mixing.

The next step in the development of this algorithm is to implement a function that allows to determine optimal dose of coagulant for specified water chemical parameters. 
Also it is possible to upgrade algorithm to simulate not only single floc formation, but simulate flocs distribution in defined volume of water.

\section{Conclusions}

1. Proposed algorithm allowed to define flocs size, volume, mass and their strength. The strength value is the main parameter, since purification effect of separation process is totally dependent on flocs strength.

2. Proposed algorithm allowed to perform modeling of possible flocs shape and allows to visualize the simulation results in $3 \mathrm{D}$ regarding to the usage of different parameters of coagulation.

3. Proposed algorithm and created computer program allowed to define the optimal parameters, as tank parameters, stirrer's parameters, mixing parameters, $\mathrm{pH}$ for carrying out coagulation.

4. Multiple simulation according to the theory of rapid Smoluchowski's coagulation allowed to obtain a number of flocs that may occur in the system, and also allowed to obtain parameters of all arising flocs.

\section{References}

1. B. Derjaguin, Prog. Surf. Sci. 43, 1-14 (1993)

2. L. Boinovich, Curr. Opin. in Colloid Interface Sci. 15, 297-302 (2010)

3. K.A. Kusters, J.G. Wijers, D. Thoenes, Chem. Eng. Sci. 52, 107-121 (1997)

4. D.N. Thomas, S.J. Judd, N. Fawcett, Wat. Res. 33, 1579-1592 (1999)

5. W. He, J. Nan, J. Song, X. Song, Int. Conf. of Energy and Environment Tech. 441-445 (2009)

6. F.E. Torres, W.B. Russel, W.R. Schowalter, J. Colloid Interface Sci. 145, 51-73 (1991)

7. R. Wardzyńska, L. Smoczyński, R. Wolicki, B. Załęska-Chróst, Z. Bukowski, Ecol. Chem. Eng. 17, 1663-1672 (2010)

8. И.Д. Сорокина, А.Ф. Дресвянников, Е.В. Петрова, А.Р. Камалиева, Вестник Казанского технологического университета, 43-45 (2015)

9. P. Jarvis, B. Jefferson, J. Gregory, S.A. Parsons, Wat. Res. 39, 3121-3127 (2005)

10. F.L. Leite, C.C. Bueno, A.L. Da Róz, E.C. Ziemath, Jr. O. N. Oliveira, Int. J. Mol. Sci. 13, 12773-12856 (2012)

11. S. Hiraoka, I. Yamada, T. Aragaki, J. Chem. Eng. Jpn. 31, 79-86 (1988) 